\title{
Limits on neutrino mixing with new heavy particles
}

\author{
Enrico Nardi ${ }^{a, 1}$, Esteban Roulet ${ }^{b, 2}$, Daniele Tommasini ${ }^{c, 3}$ \\ a Randall Laboratory of Physics, University of Michigan, Ann Arbor, MI 48109-1120, USA \\ b Theory Division, CERN, CH-1211 Geneva 23, Switzerland \\ c Departament de Fi sica Teorica, Universitat de València, and I.F.I.C. 46100 Burjassot, Valencia, Spain
}

Recelved 9 February 1994; revised manuscript received 3 March 1994

Editor: R. Gatto

\begin{abstract}
We study the effects induced by new neutral fermions below their mass threshold, due to their possible mixing with the standard neutrinos. We use as experimental constraints the recent results on lepton universality, together with the measurement of the $\mu$ decay rate and the updated LEP data. In particular, the inclusion in our data set of the most recent determinations of the $\tau$ branching fractions, mass and lifetime implies that a previous indication of a non-vanishing mixing for $\nu_{\tau}$ is no longer present. We obtain new stringent limits on the mixing parameters between $\nu_{e}, \nu_{\mu}, \nu_{\tau}$ and heavy neutral states of different weak isospin. If no assumption on the type of neutrinos involved in the mixing is made, we find $s_{\nu_{e}}^{2}<0.0071, s_{\nu_{\mu}}^{2}<0.0014$ and $s_{\nu_{\tau}}^{2}<0.033$.
\end{abstract}

\section{Introduction}

Several extensions of the Standard Model (SM), such as the Left-Right, $S O(10)$ and $\mathrm{E}_{6}$ models, predict the existence of new neutral fermions. LEP results imply that these new particles, if they are not singlets under the SM group, should be heavier than $\sim M_{Z} / 2$. Even in the case in which these new particles are too heavy to be directly produced, their presence could still affect the physics at low energy, since in general they will be mixed with the SM neutrinos. Such a mixing will affect the neutral current (NC) and charged current (CC) couplings of the known neutral states, and hence can be constrained by the precision tests of the SM. Previous model-independent, comprehen-

\footnotetext{
1 E-mail nardi@umiphys

2 E-mail: vxcern:roulet.

3 E-marl: tommasin@evalvx
}

sive analyses were performed in Refs. [1-3], resulting in particular in limits on the neutrino mixing angles roughly at the level of $\leq 10^{-1}$.

Recently, new precise tests of universality from the leptonic decays of the $\pi$ meson [4,5] and from $\tau$ decays $[6,7]$ have become available. New determinations of the $\tau$ mass [8] and lifetime [9] have also removed the previous $\sim 2 \sigma$ discrepancy from the SM predictions in $\tau$ decays ${ }^{4}$. In addition, the LEP determination of the invisible width of the $Z$ boson has also improved.

In this paper we take into account all these results, including also the experimental data on $\mu$ decay, and derive new stringent limits on the mixing angles of the

\footnotetext{
4 In a previous analysis [2] this discrepancy appeared to be an indication of a non-vanishing mixing between $\nu_{\tau}$ and new heavy neutral state with weak-isospin $+\frac{1}{2}$.
} 
known neutrinos with new neutral fermions heavier than a few $\mathrm{GeV}$.

From the theoretical point of view, these results are particularly important in order to constrain a class of models in which large mixing angles of the standard neutrinos with new states are allowed, keeping at the same time the masses of the known neutrinos below the laboratory limits. As we will discuss in Section 2 , these models require an (almost) degenerate mass matrix for the neutral states, so that vanishing (very small) masses for SM neutrinos can result in a natural way.

\section{Mixing with new heavy neutrinos}

We will describe all the new independent neutral fermionic degrees of freedom by left-handed fields arranged in a vector $\nu_{\mathcal{N}}$, without distinguishing between neutrinos and antineutrinos [1-3]. Thus we introduce a vector $n^{0 T}=\left(\nu^{T}, \nu_{\mathcal{N}}^{T}\right)$ of left-handed spinors, where $\nu^{T} \equiv\left(\nu_{e L}, \nu_{\mu L}, \nu_{\tau L}\right)$ are the known neutrinos. The fields appearing in $\boldsymbol{n}^{0}$ are gauge eigenstates. The general mass term for the neutral states is

$\mathcal{L}_{\text {mass }}=\frac{1}{2} n^{0 T} C \mathbf{M} n^{0}$,

where $C$ is the matrix of charge conjugation. The matrix $\mathbf{M}$ is symmetric and can be diagonalized through an "orthogonal" transformation $\mathbf{U}^{\mathrm{T}} \mathbf{M U}=\mathbf{M}_{\text {diag}}$, where $\mathbf{U}$ is unitary,

$\boldsymbol{n}^{0}=\mathbf{U} \boldsymbol{n}, \quad \mathbf{U}^{\dagger} \mathbf{U}=\mathbf{I}$.

We can write the vector $n$ of the mass eigenstates as $\boldsymbol{n}^{T}=\left(n^{T}, n_{h}^{T}\right)$, where $n$ are the light neutrinos and $n_{h}$ the heavy ones. Here we assume that the number of states in the vector $n$ is three, corresponding to the number of standard neutrinos measured at LEP. The matrix $\mathbf{U}$ can then be written in block form as

$\mathbf{U}=\left(\begin{array}{ll}A & G \\ F & H\end{array}\right)$

so that the unitarity conditions read $A^{\dagger} A+F^{\dagger} F=$ $A A^{\dagger}+G G^{\dagger}=I$.

We will allow for the general possibility that different kinds of new neutrinos, with different $S U$ (2) transformation properties, be present. Then the vector $\nu_{\mathcal{N}}$ can be decomposed in subspaces labelled by different values of the weak isospin $t_{3}$, $\nu_{\mathcal{N}}^{T}=\left(\nu_{t_{3}=1 / 2}^{T}, \nu_{t_{3}=0}^{T}, \nu_{t_{3}=-1 / 2}^{T}, \nu_{t_{3}=+1}^{T}, \nu_{t_{3}=-1}^{T}, \ldots\right)$. For instance, the subspace labelled by $t_{3}=0$ contains neutrinos from singlets (or from real triplets, which sometimes have been considered in the literature [10], or even from larger real multiplets). The $t_{3}=$ $1 / 2$ subspace contains new ordinary neutrinos (i.e. transforming as the standard doublet neutrinos), and the $t_{3}=-1 / 2$ subspace contains exotic neutrinos belonging to right-handed doublets that can appear in models with mirror and/or vector doublets of leptons. All these possibilities are simultaneously present, for example, in $E_{6}$ models. The above formalism allows also for other possibilities, e.g. $t_{3}= \pm 1$, corresponding to neutrinos appearing in $S U(2)_{L}$ complex triplets. Accordingly the matrices $F$ and $H$ can also be decomposed in submatrices corresponding to different subspaces, e.g. $F^{T}=\left(F_{t_{3}=1 / 2}^{T}, F_{t_{3}=0}^{T}, \ldots\right)$.

The charged current $J_{W}^{\mu}=\bar{\nu} \gamma^{\mu} e_{L}$, projected onto the subspace of the light states, reads

$J_{W}^{\mu}=\bar{n} \gamma^{\mu} A^{\dagger} e_{L}$.

In general, whenever the neutrinos mix with neutral states in non-singlet representations, we can expect that also the left-handed charged leptons will be mixed with the new charged states present in the new multiplets. This would give an additional suppression factor for the CC coupling in (4), which would result in constraints on the $\nu$ mixings stronger than the conservative limits we will obtain below. In addition to this, an induced right-handed current term would be present on the r.h.s in (4) whenever both the neutrinos and the right-handed charged leptons mix with exotic states in right-handed doublets. As discussed in detail in Refs. $[2,3]$, its effect is of higher order in the light-heavy mixings, and thus can be neglected in the present analysis. This allows us to restrict the set of new physics parameters that describe the mixing effects in the CC observables to the mixings of the neutrinos, since the corresponding constraints will still hold in the presence of non-zero mixings for charged leptons.

Denoting with $\mathbf{T}_{3}$ the generator corresponding to the third component of weak isospin, the NC coupled to the $Z$ boson is $J_{Z}^{\mu}=\bar{n}^{0} \gamma^{\mu} \mathbf{T}_{3} n^{0}=\bar{n} \gamma^{\mu} \mathbf{U}^{\dagger} \mathbf{T}_{3} \mathbf{U}$. When projected onto the light states, this gives

$J_{Z}^{\mu}=\bar{n} \gamma^{\mu}\left[\frac{1}{2} A^{\dagger} A+F^{\dagger} T_{3} F\right] n$. 
The matrix $T_{3}$ is diagonal, with entries given by the values of the weak isospin on the corresponding components of $\boldsymbol{n}^{0}$.

In processes occurring below the mass threshold for the production of the heavy states, the standard gauge eigenstate $\nu_{a}(a=e, \mu, \tau)$ is effectively replaced by its (normalized) projection $\left|\nu_{a}^{\text {light }}\right\rangle$ onto the subspace of the light neutrinos $\left|n_{t}\right\rangle(i=1,2,3)$,

$\left|\nu_{a}^{\text {light }}\right\rangle \equiv \frac{1}{c_{\nu_{a}}} \sum_{i=1}^{3} A_{\imath a}^{\dagger}\left|n_{\imath}\right\rangle$,

where $c_{\nu_{a}}^{2} \equiv \cos ^{2} \theta_{\nu_{a}} \equiv\left(A A^{\dagger}\right)_{a a}$. The state $\left|\nu_{a}^{\text {light }}\right\rangle$ has non-trivial projections on the subspace of the standard neutrinos $\left|\nu_{b}\right\rangle$ as well as on the subspaces of the new neutrinos $\left|\nu_{B}^{t_{3}}\right\rangle$, where the superscript refers to the particular value $t_{3}$ of the weak isospin. In fact we have

$\sum_{b}\left|\left\langle\nu_{b} \mid \nu_{a}^{\text {light }}\right\rangle\right|^{2}=\frac{\left(A A^{\dagger}\right)_{a a}^{2}}{c_{\nu_{a}}^{2}}=c_{\nu_{a}}^{2}$,

$\sum_{B}\left|\left\langle\nu_{B}^{t_{3}} \mid \nu_{a}^{\mathrm{lght}}\right\rangle\right|^{2}=\frac{\left(A F_{t_{3}}^{\dagger} F_{t_{3}} A^{\dagger}\right)_{a a}}{c_{\nu_{a}}^{2}} \equiv \lambda_{t_{3}}^{a} s_{\nu_{a}}^{2}$,

with $s_{\nu_{a}}^{2} \equiv 1-c_{\nu_{a}}^{2}=\sin ^{2} \theta_{\nu_{a}}$, and $\sum_{t_{3}} \lambda_{t_{3}}^{a}=1$ from unitarity. The parameter $\theta_{\nu_{a}}$ measures the total amount of mixing of the known state of flavour $a=e, \mu, \tau$ with the new states, while $\lambda_{t_{3}}^{a}$ gives the relative weight of the particular mixing involving new states of weak isospin $t_{3}$. These sets of parameters are sufficient to describe the effects of the light-heavy mixing in all the $\mathrm{CC}$ and NC processes that we will discuss. Let us consider as an example a weak decay involving the transition $e_{a} \rightarrow n_{l}$, where the subscript $a=e, \mu, \tau$ labels the flavour and $i=1,2,3$ corresponds to a light-mass eigenstate. From Eq. (4), the change with respect to the SM decay rate induced by the neutrino mixings is

$\frac{1}{\Gamma_{\mathrm{SM}}} \sum_{i} \Gamma\left(e_{a} \rightarrow n_{l}\right)=\left(A A^{\dagger}\right)_{a a}=c_{\nu_{a}}^{2}$,

and depends only on the global reduction $c_{\nu_{a}}$ in the couplings of the light neutrinos.

Before concluding this section we want to comment on the theoretical expectations for the values of the mixing parameters. In the usual see-saw mechanism for the generation of small neutrino masses, the lightheavy mixing angle $\theta$ depends on the ratio between the light and heavy mass scales, and typically we have $\sin ^{2} \theta \sim m / M$. For $M \geq M_{Z} / 2$, even if we take $m$ as large as allowed by the laboratory limit on the $\nu_{\tau}$ mass $\sim 31 \mathrm{MeV}[11]^{5}$, we get $\sin ^{2} \theta \leq 10^{-3}$, that is more than one order of magnitude smaller than the constraints that we will derive from the experimental data in Section 4.

In general it is hard to obtain larger mixing of the standard neutrinos with particles heavier than $\sim M_{Z} / 2$ without conflicting with the laboratory limits on the $\nu$ masses [11], unless the matrix $\mathbf{M}$ is (almost) degenerate. This can occur if (at least) two kinds of new states are present. For instance, let us assume that a pair $N, N^{c}$ of new neutrinos exists, with the lepton number assignments $L(N)=-L\left(N^{c}\right)=L(\nu)=$ 1 , and that $L$ is conserved. Then, in the basis $n^{T}=$ $\left(\nu^{T}, N^{T}, N^{c T}\right)$, the mass matrix is

$\mathbf{M}=\left(\begin{array}{ccc}0 & 0 & M_{\nu N^{c}} \\ 0 & 0 & M_{N N^{c}} \\ M_{\nu N^{c}} & M_{N N^{c}} & 0\end{array}\right)$,

which is degenerate, ensuring that three eigenstates form massless Weyl neutrinos. This is due to the fact that while the heavy states form Dirac neutrinos, the light states remain with no chirality partner and hence massless. However, small $L$-violating Majorana mass terms for the states $\nu$ and $N$ could also be allowed, and could be relevant for explaining the solar neutrino deficit via neutrino oscillations.

Mass matrices of the form (9) can arise for example in generalized $E_{6}$ models $[13,14]$, as well as in models predicting other kinds of vector multiplets (singlets, triplets, ...) or new mirror multiplets of leptons with neutral components $N, N^{c}$.

Clearly, if in (9) $M_{\nu N^{c}} \sim M_{N N^{c}}$, the mixing angle between $\nu$ and $N$ can be arbitrarily large. For example if the new neutrinos $N$ are ordinary, then $M_{\nu N^{c}}$ and $M_{N N^{c}}$ could be generated by vacuum expectation values of Higgs fields transforming in the same way under $S U(2)$ so that the $\nu-N$ mixing could be naturally close to maximal.

A different possibility would be to have light singlets, whose mass is not constrained by LEP and hence the see-saw suppression is less severe. Besides the con-

\footnotetext{
5 Such a large mass would require an unstable $\nu_{\tau}\left(\tau\left(\nu_{\tau}\right) \leq 40\right.$ s) not to conflict with the cosmological limits derived from big bang nucleosynthesis [12]
} 
straints that we obtain below, which hold for $m_{\nu_{s}} \geq$ $\mathrm{GeV}$, significant bounds also exist for lighter singlets $\left(m_{\nu_{s}} \ll \mathrm{GeV}\right)$ coming from primordial nucleosynthesis, since their mixing with active states can bring them, via oscillations, into equilibrium [15].

\section{Experimental constraints}

All the precise laboratory experiments are in good agreement with the predictions of the SM, which depend on a set of fundamental input parameters. Here we choose the QED coupling constant $\alpha$ measured at $q^{2}=0$, the mass of the $Z$ boson $M_{Z}=91.187 \pm 0.007$ $\mathrm{GeV}$ [16] and the Fermi constant $G_{F}$. All the relevant one-loop corrections have been taken into account in the numerical analysis.

The values of $\alpha$ and $M_{Z}$ as extracted from experiments are not affected by the mixings. In contrast, the effective $\mu$-decay constant $G_{\mu}=1.16637(2) \times$ $10^{-5} \mathrm{GeV}^{-2}$ is related to the fundamental coupling $G_{F}$ through the neutrino mixing angles [1-3],

$G_{\mu}=G_{F} c_{\nu_{e}} c_{\nu_{\mu}}$

As a consequence, all the observables that depend on the strength of the weak interactions $G_{F}$ will be affected by the mixing angles $\theta_{\nu_{e}}, \theta_{\nu_{\mu}}$. This is the case, for instance, for the Cabibbo-Kobayashi-Maskawa (CKM) matrix elements.

For our analysis we have used the $\mathrm{CC}$ constraints on lepton universality and on CKM unitarity, as well as the NC constraints from the LEP measurements at the $Z$ peak.

\subsection{Lepton universality}

The ratios $g_{e} / g_{\mu}$ and $g_{\tau} / g_{\mu}$ of the leptonic couplings to the $W$ boson, which in the SM are predicted to be unity (universality), are extracted from weak decays. From Eq. (8) we get

$\left(\frac{g_{a}}{g_{\mu}}\right)^{2}=\frac{c_{\nu_{a}}^{2}}{c_{\nu_{\mu}}^{2}}, \quad a=e, \tau$

The best test of $e-\mu$ universality comes from $\pi \rightarrow e \nu$ compared to $\pi \rightarrow \mu \nu$. The ratio $R_{\pi} \equiv$ $\Gamma(\pi \rightarrow e \nu+\pi \rightarrow e \nu \gamma) / \Gamma(\pi \rightarrow \mu \nu+\pi \rightarrow \mu \nu \gamma)$ has been recently measured with great accuracy at TRIUMF, $R_{\pi}=[1.2265 \pm 0.0034$ (stat) \pm 0.0044 (syst)] $\times 10^{-4}$ [4], and at the PSI, $R_{\pi}=$ $[1.2346 \pm 0.0035$ (stat) \pm 0.0036 (syst) $] \times 10^{-4}$ [5]. Combining systematic and statistical errors in quadrature, the average of the two experiments is $R_{\pi}=[1.2310 \pm 0.0037] \times 10^{-4}$, to be compared with the theoretical SM prediction $R_{\pi}^{\mathrm{SM}}=$ [1.2352 \pm 0.0005$] \times 10^{-4}$ [17]. Thus we obtain $\left(g_{e} / g_{\mu}\right)^{2}=R_{\pi} / R_{\pi}^{\mathrm{SM}}=0.9966 \pm 0.0030$.

The best tests of $\mu-\tau$ universality come from the $\tau$ leptonic decays compared to $\mu$ decay. The new world average, including LEP data, gives $\left(g_{\tau} / g_{\mu}\right)^{2}=$ $0.989 \pm 0.016[7]$. This value takes into account also the recent improvements on the determinations of the $\tau$ mass [8] and lifetime [9], and is no longer in conflict with the SM prediction of unity. A second test comes from $\tau \rightarrow \pi(K) \nu_{\tau}$, which gives $\left(g_{\tau} / g_{\mu}\right)^{2}=$ $1.051 \pm 0.029$ [7]; this is almost $2 \sigma$ off the SM, and hardly compatible with the above determination from $\tau$ decays. In our analysis, we have used the first, more accurate determination of $\left(g_{\tau} / g_{\mu}\right)^{2}$, which is also theoretically clearer. However, we will also give the results obtained including both these constraints at the same time.

\subsection{CKM unitarity}

The observed CKM matrix elements $V_{u d}$ and $V_{u s}$ are obtained by dividing by $G_{\mu}$ the measured vector coupling in $\beta$ decay and in $K_{e 3}$ and hyperon decays, respectively. These processes are weak decays involving a vertex with $\nu_{e}$, so that a factor $c_{\nu_{e}}$ is present. Then the effect of the mixing is to modify $V_{u l}, i=d, s$, by a factor $c_{\nu_{e}} G_{F} / G_{\mu}[1,2]$. The value of $\left|V_{u b}\right|$, obtained from the analysis of semileptonic $B$ decays, is negligibly small for our purposes. Then the unitarity constraint for the first row of the CKM matrix in the presence of neutrino mixing becomes [1,2]

$\sum_{i=1}^{3}\left|V_{u i}\right|^{2}=\left(\frac{G_{F}}{G_{\mu}} c_{\nu_{e}}\right)^{2}$.

Due to Eq. (10), this depends only on $s_{\nu_{\mu}}$. This theoretical expression has to be compared with the result extracted from experiments [18]: $\sum_{t=1}^{3}\left|V_{u t}\right|^{2}=$ $0.9992 \pm 0.0014$. 


\subsection{Invisible width of the $Z$ boson}

The invisible decay rate of the $Z$ boson into neutrinos is proportional to the Fermi constant $G_{F}$ multiplied by the sum of the squares of the neutrino NC couplings in (5). Normalizing to the SM contribution, and keeping only the first order in the light-heavy mixing, we get

$$
\begin{gathered}
\frac{\Gamma_{Z \rightarrow \mathrm{nv}}}{\Gamma_{Z \rightarrow \mathrm{Inv}}^{\mathrm{SM}}}=\frac{G_{F}}{G_{\mu}} \frac{\operatorname{Tr}\left(A^{\dagger} A+2 F^{\dagger} T_{3} F\right)^{2}}{3} \\
\simeq \frac{G_{F}}{G_{\mu}}\left[1-\frac{1}{3} \sum_{a} \Lambda_{a} s_{\nu_{a}}^{2}+O\left(s^{4}\right)\right],
\end{gathered}
$$

where the parameters $\Lambda^{a} \equiv 2 \sum_{t_{3}}\left(1-2 t_{3}\right) \lambda_{t_{3}}^{a}$ describe the particular admixture of heavy neutrinos, assumed heavier than $M_{Z} / 2$, involved in the mixing.

If the light states are mixed only with new ordinary states, corresponding to $\lambda_{t_{3}=1 / 2}=1(\Lambda=0)$, the invisible width is slightly increased by the factor $G_{F} / G_{\mu}$. A mixing with singlet neutrinos $\left(\lambda_{t_{3}=0}=1, \Lambda=2\right)$ or with neutrinos from exotic doublets $\left(\lambda_{t_{3}}=-1 / 2=1\right.$, $\Lambda=4$ ) will give a reduction in the effective number of neutrinos. Neutrinos appearing in different kinds of lepton triplets behave in different ways. For real triplets the neutral component has $t_{3}=0$ and behaves essentially like a singlet. Neutrinos in complex triplets have $t_{3}= \pm 1$, so that $\Lambda=-2$ or $\Lambda=6$.

We see that the effects of light-heavy neutrino mixing can be described by means of the parameters $\lambda_{t_{3}}^{a}$ (appearing in the three combinations $\Lambda_{a}$ ) and $\theta_{\nu_{a}}$, which together define the decomposition (6) of the vector $\left|\nu_{a}^{\text {light }}\right\rangle$.

As we discussed in Section 2, in the presence of mixings in the neutral sector it is natural to expect that also the charged leptons will be mixed with new heavy partners, implying a possible violation of universality for their couplings to the $Z$ boson. We then use, for consistency, a determination of $\Gamma_{Z \rightarrow 1 \mathrm{nv}}$ from the LEP experiments, which is independent of the assumption of universality for the charged-lepton couplings. Let us consider the flavour-dependent $Z$ line-shape parameters $\Gamma_{Z}, \sigma_{h}^{0}, R_{e}, R_{\mu}, R_{\tau}$ [16], where $\Gamma_{Z}$ is the total width of the $Z$ boson, $\sigma_{h}^{0}$ is the hadronic cross section at the peak, and $R_{l} \equiv \Gamma_{h} / \Gamma_{l}$ are the ratios of the hadronic to the leptonic $(l=e, \mu, \tau)$ partial widths. We can define $\Gamma_{Z \rightarrow \text { nv }}=\Gamma_{Z}-\Gamma_{h}-\sum_{l=e, \mu, \tau} \Gamma_{l}$. Then from the relation $\sigma_{h}^{0}=\frac{12 \pi}{M_{Z}^{2}} \frac{\Gamma_{h} \Gamma_{c}}{\Gamma_{Z}^{2}}$ we get

$$
\Gamma_{Z \rightarrow \mathrm{nv}}=\Gamma_{Z}\left[1-\sqrt{\frac{M_{Z}^{2}}{12 \pi} R_{e} \sigma_{h}^{0}}\left(1+\sum_{l} \frac{1}{R_{l}}\right)\right] .
$$

Using the experimental determinations [16] for the set $\Gamma_{Z}, \sigma_{h}^{0}, R_{e}, R_{\mu}, R_{\tau}$ and its correlation matrix, we obtain $\Gamma_{Z \rightarrow \mathrm{nv}}=(498.6 \pm 5.2) \mathrm{MeV}$, slightly different from the value obtained in Ref. [16] with the assumption of universality. Taking into account the SM prediction $\Gamma_{Z \rightarrow \text { nv }}=3\left(G_{\mu} M_{Z}^{3} / 12 \sqrt{2} \pi\right) \rho=$ (497.6 MeV) $\rho$, with $\rho \simeq 1+\frac{3}{8 \sqrt{2} \pi^{2}} G_{\mu} m_{t}^{2}$, we finally obtain $\Gamma_{Z \rightarrow \mathrm{nv}} / \Gamma_{Z \rightarrow \mathrm{nv}}^{\mathrm{SM}}=(1.002 \pm 0.010) / \rho$. The dependence on $\rho$ will give a correlation between the top quark mass $m_{t}$ and the neutrino mixing angles (mainly $s_{\nu_{r}}$, which is less constrained by other measurements).

Besides the constraints arising from the invisible width, Eq. (13), obtained under the assumption $M>M_{Z} / 2$, an important signature at the $Z$ peak of fermionic mixing is the single production of a heavy state (provided it is lighter than $M_{Z}$ ) in association with a light one. In this case, visible signals due to the decay of the heavy neutrino could be present $[19,20]$, since the mixing will also imply that the heavy neutrino can decay through the channels $n_{h} \rightarrow$ $\nu+Z^{*} \rightarrow \ell^{+} \ell^{-}, q \bar{q}$ and $n_{h} \rightarrow \ell+W^{*} \rightarrow \ell \nu_{\ell}^{-}, q q^{\prime}$. A search for signals of these decays has been performed at LEP for heavy singlets in the mass range $3 \mathrm{GeV}$ $<M<M_{Z}[21,22]$. For $3 \mathrm{GeV}<M<50 \mathrm{GeV}$ the resulting limits, $s_{\nu_{a}}^{2} \leq 10^{-4}$, are very stringent, and they worsen for $M$ approaching $M_{Z}$ due to phase space suppression of the production rate [22]. This mechanism provides then a tool to search for tiny mixing angles with states lighter than $M_{Z}$, a search that is complementary to the ones discussed in this paper.

\section{Results}

We have collected all the theoretical predictions and the experimental results for the electroweak observables in a $\chi^{2}$ function. We have verified that the zero mixing case $\left(s_{\nu_{a}}=0\right.$ for $\left.a=e, \mu, \tau\right)$ lies inside the $90 \%$ confidence level region, so that there are no evidences for neutrino mixing with new particles. The former $2 \sigma$ disagreement of the data from $\tau$ decays has 
Table 1

Value of the $90 \%$ c.l upper bound on $s_{\nu_{\tau}}^{2}$, corresponding to a set of values of the parameter $\Lambda_{\tau}$ describing the type of the new heavy particles involved in the mixing with $\nu_{\tau}$

\begin{tabular}{llllll}
\hline & \multicolumn{1}{c}{$\Lambda_{\tau}$} & & & & \\
\cline { 2 - 6 } & 0 & 2 & 4 & 6 & -2 \\
\hline Conservative bound & 0.033 & 0.024 & 0.015 & 0010 & 0.019 \\
Using $\tau \rightarrow \pi(K) \nu_{\tau}$ & 0.020 & 0017 & 0012 & 0.0089 & 0.013 \\
\hline
\end{tabular}

The top mass has been fixed to $m_{t}=150 \mathrm{GeV}$ The first line gives the conservative limits, obtained using the set of constraints including the data on $\mu$ and $\tau$ leptonic decays and on the invisible width of the $Z$ boson. The tighter bounds given in the second line are less conservative, since they have been obtained by including in the data set also the constraint from $\tau \rightarrow \pi(K) \nu_{\tau}$.

disappeared, owing to the new experimental results discussed in Section 3. As a result, also the signal of a possible non-zero mixing of the $\tau$ neutrinos with new ordinary states, which was found in a previous analysis [2], is no longer present. Since no signals of nonzero mixings are found, all the results of our fit are presented as ( $90 \%$ c.l.) upper bounds on the mixing angles.

For the limits on the mixings $s_{\nu_{e}}^{2}$ and $s_{\nu_{\mu}}^{2}$ we obtain

$s_{\nu_{e}}^{2}<0.0071, \quad s_{\nu_{\mu}}^{2}<0.0014$,

almost independently of $\Lambda_{e}$ and $\Lambda_{\mu}$. This is due to the fact that the main sources of constraints are CC processes, namely $\mu$ decay and the lepton universality test on $\left(g_{e} / g_{\mu}\right)^{2}$ for $s_{\nu_{e}}^{2}$, and the CKM unitarity test (depending on the $\mu$-decay constant) for $s_{\nu_{\mu}}^{2}$. The constraints from the LEP data are much less stringent than the previous ones, and for this reason the limits (15) will not change significantly, even if the new neutral states involved in the mixing were lighter than $M_{Z}$. However they are still assumed to be heavier than the production energy thresholds in the relevant processes used for the experimental determinations, that is $m_{\pi}$ and $m_{\Lambda}$ respectively.

In contrast, the limits on $s_{\nu_{\tau}}^{2}$ do depend on the value of $\Lambda_{\tau}$, that is on the weak isospin of the new neutrinos involved in the mixing. The different limits are given in Table 1 . The conservative bounds are obtained using only the determination of the universality ratio from $\tau$ decays and the results from LEP. In the second line we give the more stringent, but less conservative, bounds obtained by including in the data set the constraint from $\tau \rightarrow \pi(K) \nu_{r}$. The result for $\Lambda_{\tau}=0$ coincides also with the bound that can be set using only the CC constraints, since for this particular value of $\Lambda_{\tau}$ the NC couplings are not affected by $s_{\nu_{\nu^{*}}}^{2}$ This bound,

$$
\begin{aligned}
s_{\nu_{\tau}}^{2} & <0.033 \\
& \left(0.020 \text { including } \tau \rightarrow \pi(K) \nu_{\tau}\right),
\end{aligned}
$$

is valid for any $\Lambda_{\tau}$ provided that the new particles involved in the mixing are heavier than $m_{\tau}$, and improves by a factor $3(5)$ the previous result $\left(s_{\nu_{\tau}}^{2}<0.098\right.$ for $\Lambda_{\tau}=0$ [2]). For $\Lambda_{\tau} \neq 0$ the bounds are more stringent, due to the effectiveness of the additional constraint from the $Z$ invisible width. Assuming that the new particles are heavier than $M_{Z}$, and taking the value $m_{t}=150 \mathrm{GeV}$ for the top-quark mass, this constraint alone sets a limit $s_{\nu_{r}}^{2}<0.060 / \Lambda_{\tau}$ for $\Lambda_{\tau}>0$ $\left(s_{\nu_{r}}^{2}<0.040 /\left|\Lambda_{\tau}\right|\right.$ for $\left.\Lambda_{\tau}<0\right)$. For $\left|\Lambda_{\tau}\right| \geq 1$ this is comparable to the effect of the CC constraint, and the combined result is given in the table. For large $|\Lambda|$ the constraint from LEP data is the most stringent though somewhat less general, since it holds only under the assumption that the new particles involved in the mixing are heavier than $M_{Z}$. The bounds change slightly (at most by $\sim 20 \%$ of their value) for different choices of $m_{t}$ in the range $110 \mathrm{GeV}<m_{t}<200 \mathrm{GeV}$. In general, since $\Gamma_{Z \rightarrow \mathrm{nv}} \sim\left(1-\frac{1}{3} \Lambda_{\tau} s_{\nu_{\tau}}^{2}\right)\left(1+\frac{3}{8 \sqrt{2} \pi^{2}} G_{\mu} m_{t}^{2}\right)$ the limit is weakened either for greater $m_{t}$ if $\Lambda_{\tau}>0$, or for smaller $m_{t}$ when $\Lambda_{\tau}<0$.

As we have already mentioned, models predicting new neutral fermions often contain also additional charged fermions as well as new neutral gauge bosons $[2,3]$. Then in some observables the contributions due to these additional sources of new physics can compensate in part the effects of the light-heavy neutrino mixing. As we have discussed, our limits are still reliable in the presence of non-zero mixings for the charged leptons. However, as is shown in Refs. [2,3], the additional effects of new neutral gauge bosons and especially of non-zero light-heavy mixings in the quark sector can relax the bounds on the neutrino mixing angles. The most important cancellations can affect the bound on $s_{\nu_{\mu}}^{2}$, whose contribution to the CKM unitarity sum can be compensated by a mixing between the left-handed quarks and new exotic particles [1-3]. 


\section{Conclusions}

We have studied the effects induced by new neutral fermions below the threshold for their direct production. The possible mixing of these new particles with the standard neutrinos would affect the NC and CC processes for the light states as discussed in Section 2. The CC experimental test on lepton universality and $\mu$ decay, as well as the NC data on the $Z$ boson invisible width, have been used to constrain the light-heavy neutrino mixing angles. We have found no evidence for this kind of new physics effects, and the new limits we have obtained on the mixing parameters improve the results of previous analyses [1-3]. We have also generalized our discussion to include new states of arbitrary weak isospin. Our main results are summarized by Eqs. (15) and (16). These limits are general in the sense that they hold independently of the weak isospin of the new states involved in the mixing. The underlying assumption that the new states are heavier than $m_{\pi}, m_{\Lambda}, m_{\tau}$, respectively, for the neutrino mixings of the first, second and third generation, is non-trivial only for the mixing with singlet neutrinos (or more in general, neutrinos with third component of weak isospin $t_{3}=0$ ). In the particular case of $s_{\nu_{r}}$, the limits can be more stringent depending on the specific value assumed for the weak isospin of the heavy states. The constraints from the LEP measurement of the invisible width is very important in this case. A list of limits that correspond to different possible values of the weak-isospin for the new states is given in Table 1.

Our bounds are of little use in the framework of see-saw models, which in fact predict light-heavy mixing angles much smaller than the experimental limits. However, different models exist in which the mixing angles between the known and the new heavy neutral states can be naturally large, and they are directly constrained by our results. For example we have shown that this is the case for a class of models that predict an (almost) degenerate mass matrix in the neutral sector, since in this case large mixing angles are naturally consistent with vanishing (small) masses for the standard neutrinos.

\section{Acknowledgement}

We would like to thank E. Akhmedov and J. Valle for useful discussions.

\section{References}

[1] P. Langacker and D. London, Phys. Rev D 38 (1988) 886.

[2] E Nard1, E. Roulet and D. Tommasini, Nucl Phys. B 386 (1992) 239.

[3] E. Nardi, E Roulet and D. Tommasinı, Phys. Rev. D 46 (1992) 3040

[4] D.I. Britton et al, Phys Rev. Lett. 68 (1992) 3000.

[5] G Czapek et al., Phys. Rev. Lett. 70 (1992) 17.

[6] N. Colino (L3), in Proc. of the Second Workshop on Tau Lepton Physics ed. K K Gan (1992) (World Scientific, Singapore, 1993);

S Snow (ALEPH), ibidem;

J Hobbs (OPAL), 1bidem;

P. Vaz (DELPHI), ibidem,

N. Mistry (CLEO II), ibidem

[7] J.M. Roney, in Proc. of the Second Workshop on Tau Lepton Physics ed K.K. Gan (1992) (World Scientific, Singapore, 1993).

[8] CLEO Collab., R Ballest et al., prepnnt CLNS-93-1194, BEPC Collab, J.Z. Bai et al., Phys. Rev. Lett 69 (1992) 3021;

D. Brtton, in Proc. of the Second Workshop on Tau Lepton Physics ed. K.K Gan (1992) (World Scientific, Singapore, 1993),

H Marsiske, ibidem.

[9] ALEPH Collab., Phys Lett B 279 (1992) 411; Phys. Lett. B 297 (1992) 432,

OPAL Collab, Z Phys. C 59 (1993) 183;

DELPHI Collab., preprint CERN-PPE/93-12 (1993);

M. Uzerman (L3 Collab.), in Proc. of the Second Workshop on Tau Lepton Physics ed. K.K. Gan (1992) (World Scientific, Singapore, 1993);

W. Trischuk, ibıdem; CLEO Collab, M Battle et al., Phys. Lett. B 291 (1992) 488.

[10] B.W Lee, Phys Rev. D 6 (1972) 1188;

J Prentki and B. Zumino, Nucl. Phys B 47 (1972) 99;

P Salati, Phys. Lett. B 253 (1991) 173.

[11] Particle Data Group, Phys Rev. D 45 (1992) 1.

[12] E Kolb, M.S. Turner, A Chakravorty and D.N. Schramm, Phys Rev. Lett. 67 (1991) 553;

A D. Dolgov and I.Z. Rothstein, Phys Rev. Lett. 71 (1993) 476;

M Kawasaki et al., Report OSU-TA-5/93 and UPR-0562T (1993).

[13] R N. Mohapatra and J WF. Valle, Phys. Rev D 34 (1986) 1642 ,

J. Bernabéu et al, Phys. Lett. B 187 (1987) 303.

[14] E Nard,, Phys Rev. D 48 (1993) 3277. 
[15] R. Barbieri and A Dolgov, Phys Lett B 237 (1990) 440; X Shi et al., Phys. Rev. D 47 (1993) 3720.

[16] The LEP Collaborations and The LEP Electroweak Working Group, preprint CERN-PPE/93-157 (1993)

[17] W.J Marciano and A. Sirlin, preprint NYU-TH-93-09-03 (1993).

[18] A. Surlin, in Precision Test of the Standard Electroweak Model (World Scientific, Singapore, 1993), ed. P. Langacker.
[19] M. Gronau et al., Phys Rev. D 29 (1984) 2539.

[20] M. Dittmar, A Santamaria, M.C. Gonzalez Garcia and J.W.F. Valle, Nucl. Phys. B 332 (1990) 1.

[21] OPAL Collab., M.Z. Akrawy et al., Phys Lett. B 247 (1990) 448.

[22] L3 Collab., O Adriani et al., Phys. Lett. B 295 (1992) 371. 